BMJ Open

Diabetes

Research

\& Care

\title{
Lower extremity MRI following 10-week supervised exercise intervention in patients with diabetic peripheral neuropathy
}

Ryan Brown (D , ${ }^{1}$ Azadeh Sharafi, ${ }^{1}$ Jill M Slade, ${ }^{2}$ Antonio Convit, ${ }^{3,4}$ Nathan Davis, ${ }^{5}$ Steven Baete, ${ }^{1}$ Heather Milton, ${ }^{6}$ Kenneth J Mroczek, ${ }^{7}$ Patricia M Kluding, ${ }^{8}$ Ravinder R Regatte, ${ }^{1}$ Prodromos Parasoglou, ${ }^{1}$ Smita Rao ${ }^{9}$

To cite: Brown R, Sharafi A Slade JM, et al. Lower extremity MRI following 10week supervised exercise intervention in patients with diabetic peripheral neuropathy. BMJ Open Diab Res Care 2021;9:e002312. doi:10.1136/ bmjdrc-2021-002312

- Additional supplemental material is published online only. To view, please visit the journal online (http://dx.doi. org/10.1136/bmjdrc-2021002312).

Received 5 April 2021 Accepted 17 August 2021

Check for updates

(c) Author(s) (or their employer(s)) 2021. Re-use permitted under CC BY-NC. No commercial re-use. See rights and permissions. Published by BMJ.

For numbered affiliations see end of article.

Correspondence to Dr Ryan Brown;

Ryan.Brown@nyulangone.org

\section{ABSTRACT}

Introduction The purpose of this study was to characterize using MRI the effects of a 10-week supervised exercise program on lower extremity skeletal muscle composition, nerve microarchitecture, and metabolic function in individuals with diabetic peripheral neuropathy (DPN).

Research design and methods Twenty participants with DPN completed a longitudinal trial consisting of a 30-day control period, during which subjects made no change to their lifestyle, followed by a 10 -week intervention program that included three supervised aerobic and resistance exercise sessions per week targeting the upper and lower extremities. The participants' midcalves were scanned with multinuclear MRI two times prior to intervention (baseline ${ }_{1}$ and baseline ${ }_{2}$ ) and once following intervention to measure relaxation times (T1, $\mathrm{T} 1 \rho$, and $\mathrm{T} 2$ ), phosphocreatine recovery, fat fraction, and diffusion parameters.

Results There were no changes between baseline ${ }_{1}$ and baseline ${ }_{2}$ MRI metrics $(p>0.2)$. Significant changes $(p<0.05)$ between baseline ${ }_{2}$ and postintervention MRI metrics were: gastrocnemius medialis (GM) T1 $-2.3 \% \pm 3.0 \%$ and soleus T2 $-3.2 \% \pm 3.1 \%$. Trends toward significant changes $(0.05<p<0.1)$ between baseline ${ }_{2}$ and postintervention MRI metrics were: calf adipose infiltration $-2.6 \% \pm 6.4 \%$, GM T1 $\rho-4.1 \% \pm 7.7 \%$, GM T2 $-3.5 \% \pm 6.4 \%$, and gastrocnemius lateral T2 $-4.6 \pm 7.4 \%$. Insignificant changes were observed in gastrocnemius phosphocreatine recovery rate constant $(p>0.3)$ and tibial nerve fractional anisotropy $(p>0.6)$ and apparent diffusion coefficient $(p>0.4)$.

Conclusions The 10-week supervised exercise intervention program successfully reduced adiposity and altered resting tissue properties in the lower leg in DPN. Gastrocnemius mitochondrial oxidative capacity and tibial nerve microarchitecture changes were not observed, either due to lack of response to therapy or to lack of measurement sensitivity.

\section{INTRODUCTION}

Approximately 34 million people have diabetes in the USA, ${ }^{1}$ and $30 \%-50 \%$ of patients with type 2 diabetes develop diabetic peripheral neuropathy (DPN). ${ }^{2} \mathrm{DPN}$ is characterized by

\section{Significance of this study}

What is already known about this subject?

- Pharmacological options to treat or reverse the progression of diabetic peripheral neuropathy (DPN) are not available. While exercise has been shown to improve pain interference, vascular health, and neuropathic symptoms, muscle composition following short-term intervention has yet to be reported.

What are the new findings?

10-week supervised exercise intervention reduced MRI relaxation times in calf muscles.

> Changes in MRI relaxation times were associated with changes in clinical markers.

- 10-week supervised exercise intervention reduced adiposity in the combined calf interstitial and muscle space but not in individual calf muscles.

How might these results change the focus of research or clinical practice?

$>$ Our study shows that short-term exercise intervention improves muscle composition in DPN, which may provide clinicians with an appealing treatment option that can complement pharmaceutical intervention.

metabolic and microvascular impairment that damage peripheral nerves and cause ischemic conditions and muscle degeneration in the lower extremities. ${ }^{3}$ Prolonged DPN causes significant skeletal muscle deficits, including increased adiposity and loss of strength and endurance. ${ }^{4-6}$

Pharmacological options to treat or reverse the progression of DPN are not available and alternatively target pain response. ${ }^{7-9}$ Exercise training is an appealing option to potentially delay or reverse DPN progression because of its promise for improving glycemic control, insulin sensitivity, and adiposity in individuals with diabetes. ${ }^{10-13}$ Among interventions 
such as exercise therapy, electrotherapy, and other physiotherapy methods, exercise therapy has been the most effective. ${ }^{14}$ Studies have shown that supervised exercise improves cardiorespiratory function, innervation, ${ }^{15-17}$ nerve conduction, ${ }^{18}$ muscle strength, balance, ${ }^{19}$ and reduces pain interference. ${ }^{20}$ However, the effects of exercise on key DPN markers such as lower extremity muscle adiposity, nerve microarchitecture, and muscle metabolism have yet to be reported. MRI is an appropriate means to measure such markers, as proton-based Dixon MRI is well established for quantifying adipose content, ${ }^{46}$ diffusion tensor imaging for assessing nerve integrity, ${ }^{21}$ while dynamic phosphorus and static proton MRI, respectively, provide information on mitochondrial function ${ }^{22} 23$ and muscle tissue composition. ${ }^{24-29}$ Therefore, the goals of this longitudinal, prospective study were to determine with multinuclear MRI whether short-term supervised exercise intervention improves lower extremity adipose infiltration, neuronal microarchitecture, skeletal muscle tissue composition, and muscle oxidative capacity.

\section{RESEARCH DESIGN AND METHODS}

\section{Study design and participants}

Individuals aged between 40 and 70 years with type 2 diabetes and diabetic neuropathy or with type 2 diabetes and symptoms such as lack of monofilament and vibration perception were invited to enroll in this longitudinal study. The exclusion criteria were: difficulty walking without assistance, foot ulcer within 3 months of enrollment, partial foot amputation, stroke, central nervous system pathology, stage 2 hypertension, acute medical conditions that were deemed incompatible with exercise intervention such as cardiac failure, peripheral arterial disease, alcoholism, chronic drug use, chronic gastrointestinal disease, renal or hepatic impairment, pregnancy, and contraindications for MRI (eg, body mass index (BMI) $>40 \mathrm{~kg} / \mathrm{m}^{2}$, claustrophobia, pacemaker, or other MRI incompatible implants). DPN status was confirmed prior to MRI using the Michigan Neuropathy Screening Instrument (MNSI) $;{ }^{30}$ participants with MNSI physical exam score $<1$ were excluded. Participants received normal medical care prescribed by their physicians during the study. Recruitment began in November 2018 and was suspended in March 2020 due to restrictions related to COVID-19. Subjects participated in examinations and exercise intervention in the following chronology: week 0: baseline ${ }_{1}$ MRI and clinical tests; week 3: baseline ${ }_{2}$ MRI and aerobic fitness measurements; weeks 4-14: exercise intervention; week 15: postintervention MRI, clinical tests, and aerobic fitness measurements. Baseline, ${ }_{2}$ MRI was carried out to potentially detect changes that reflect inadvertent lifestyle adjustment following study enrollment.

\section{Clinical tests}

Blood draws were administered at baseline ${ }_{1}$ and postintervention to record glycosylated hemoglobin (HbAlc), $\mathrm{C}$ reactive protein and glucose levels at $30 \mathrm{~min}$ intervals during an oral glucose tolerance test (OGTT). The OGTT data were used to calculate the Matsuda index and homeostasis model assessment of insulin resistance (HOMA-IR). Participants were instructed to forego diabetes medications 1 day prior and fast 8-12 hours prior to blood draws.

\section{Aerobic fitness}

Peak heart rate, rating of perceived exertion, blood pressure, and peak oxygen consumption ( $\mathrm{VO}_{2}$ peak) were assessed during a treadmill test administered at baseline and postintervention. The test was performed according to the Balady Modified Ramp Protocol, ${ }^{31}$ aiming for age predicted heart rate maximum plateau and subject reported volitional exhaustion. Twelve-lead ECGs were monitored throughout exercise to screen for ischemic changes ( $\geq 1 \mathrm{~mm}$ horizontal or down-sloping ST-segments in an individual lead) and significant ectopy (three consecutive heartbeats with ventricular ectopy or onset of atrial fibrillation or tachyarrythmia).

\section{Exercise intervention}

The 10-week personalized exercise intervention program consisted of three sessions per week supervised by a physical therapist, which included moderate-intensity aerobic and resistance components. Moderate-intensity aerobic exercise $\left(50 \%-70 \%\right.$ of oxygen uptake $\left(\mathrm{V}_{2}\right)$ reserve) was performed on a treadmill or recumbent stepper and individually prescribed based on heart rate response to the graded maximum exercise test described previously. The aerobic exercise progressed from 30 to $50 \mathrm{~min}$ per session over the duration of the program. Resistance exercises included bicep and tricep curls, bent rows, leg press and standing heel raises. The resistance level for a given exercise was progressed every 3 weeks if the participant was able to complete the prescribed number of repetitions with lower rate of perceived exertion. Blood glucose, blood pressure, heart rate, rate of perceived exertion, and adverse events were monitored at each session. A visual foot examination was performed once each week.

\section{Multinuclear MRI}

All MRI experiments were performed on a 3 Tesla scanner (Prisma, Siemens Medical Solutions, Erlangen, Germany) with an in-house developed, investigational multinuclear (proton/phosphorus) lower extremity coil. ${ }^{32}$ The scans were centered at the widest lateral midcalf dimension of the right leg at baseline. The distance between the base of the foot and midcalf was measured using a ruler affixed to the system and subsequently used to landmark participants to reduce longitudinal position variability in baseline ${ }_{2}$ and postintervention scans.

To measure fat fraction, we acquired Dixon-based proton three-dimensional gradient echo data with the following parameters: echo time $(\mathrm{TE})=2.1,2.8$ and $3.7 \mathrm{~ms}$; repetition time $(\mathrm{TR})=12 \mathrm{~ms}$, field of view $(\mathrm{FOV})=220 \times 220 \times 200 \mathrm{~mm}^{3}$, resolution $=1.7 \times 1.7 \times 5.0 \mathrm{~mm}^{3}$, 
slices $=40$, and total acquisition time $=3 \mathrm{~min}$. The flip angle was set to $3^{\circ}$ to minimize $\mathrm{T} 1$ bias. ${ }^{33}$ The proton density fat fraction maps were calculated by processing the gradient echo data using the Hierarchiacal IDEAL method in MATLAB (R2019a, The MathWorks, Natick, Massachusetts, USA). ${ }^{34}$

We measured proton relaxation times at rest using a two-dimensional magnetic resonance fingerprinting technique that encoded spin-lattice relaxation (T1), spin-spin relaxation (T2), and spin-lattice relaxation in the rotating frame $(\mathrm{T} 1 \rho) .{ }^{35}$ Four consecutive axial images were acquired with FOV $=140 \times 140 \mathrm{~mm}^{2}$, resolution $=0.6 \times 0.6 \times 4.0 \mathrm{~mm}^{3}$, TE/TR=3.5/7.5 ms, BW=420 Hz/ pixel, number of shots $=4$, spin-lock power $f_{s l}=500 \mathrm{~Hz}$, and acquisition time $=4: 45 \mathrm{~min}$. The measured signal fingerprint was compressed using singular value decomposition and matched using an iterative approach to simulated MR fingerprints for possible T1, T2, T1 $\rho$, and $\mathrm{B}_{1}^{+}$values, which were computed using extended phase graphs. All processing was performed using a MATLAB script written in-house.

Fractional anisotropy (FA) and apparent diffusion coefficient (ADC) were measured in the tibial nerve (TN) using a diffusion tensor imaging (DTI) sequence with the following parameters: TR/TE $=5300 / 65 \mathrm{~ms}$, resolution $=0.5 \times 0.5 \times 3.0 \mathrm{~mm}^{3}$, image matrix $=128 \times 128$, FOV $=160 \mathrm{~mm}$, b-value $=0$ and 1200 , number of diffusion encoding directions $=12$, number of slices $=60$, and acquisition time $=5: 17 \mathrm{~min}$.

Following the resting measurements previously, we measured the phosphocreatine recovery rate time constant using a dynamic three-dimensional ${ }^{31} \mathrm{P}$-MRI FLORET (non-Cartesian Fermat looped, orthogonally encoded trajectories) pulse sequence. ${ }^{36}$ The time constant was calculated from data acquired following an exercise routine in which participants performed plantar flexion for $90 \mathrm{~s}$ at $0.66 \mathrm{~Hz}$ according to an acoustic cue on an in-house developed, MR-compatible ergometer. ${ }^{37}$ During plantar flexion, the participant moved the ergometer footplate through approximately $30^{\circ}$ range of motion with resistance applied by rubber tubing designed to deplete phosphocreatine by $15 \%-40 \%$ from its value at rest. Images with the following parameters were acquired serially for $420 \mathrm{~s}$ : $30 \mathrm{~s}$ baseline reference before the plantar flexion routine, during the 90 s routine, and $300 \mathrm{~s}$ after the routine. The following imaging parameters were used: $\mathrm{FOV}=550 \mathrm{~mm}$ isotropic, $\mathrm{TR}=0.5 \mathrm{~s}$, flip angle $=25^{\circ}, 3$ hubs at $45^{\circ}, 4$ interleaves per hub, $17 \mathrm{~mm}$ nominal isotropic resolution, 32 slices, and $6 \mathrm{~s}$ acquisition time per image.

\section{Statistical analysis}

To investigate fat fraction distribution, we manually segmented five axial slices $(25 \mathrm{~mm})$ into muscle compartments: gastrocnemius medialis (GM), gastrocnemius lateralis (GL), and soleus (SOL). Secondarily, we created an 'interior' region of interest to quantify fat fraction in the merged muscle and interstitial regions; the interior

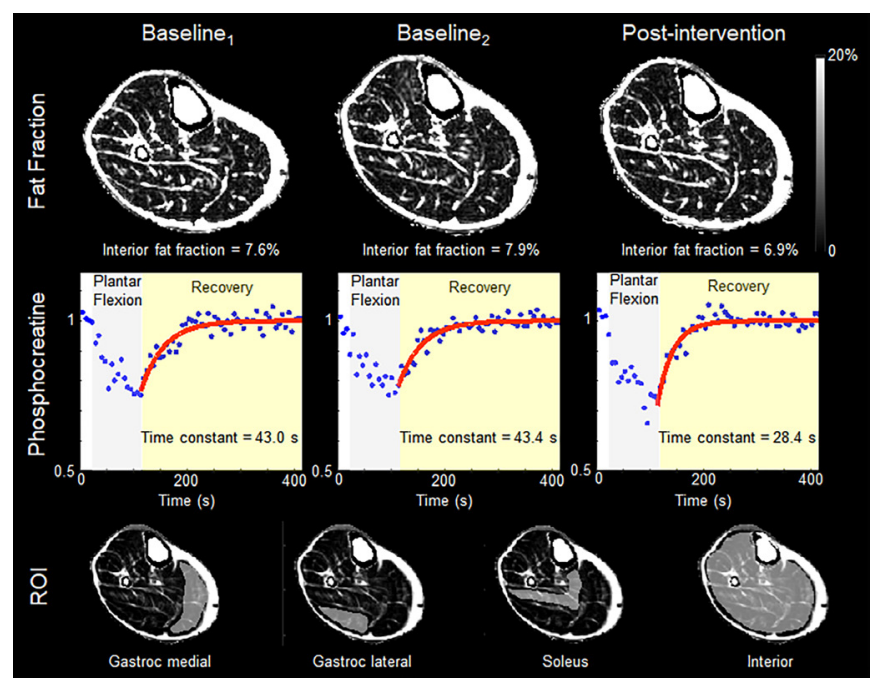

Figure $1 \mathrm{MRI}$ data from a DPN patient in their 50 s at baseline $_{1}$ (first column), baseline ${ }_{2}$ (middle column), and postintervention (last column). Row 1 shows fat fraction maps and values in the interior ROI, row 2 shows measured gastrocnemius phosphocreatine levels (blue) and fitted mono-exponential resynthesis curves (red) and time constants during recovery following plantar flexion exercise, and row 3 shows ROls. Similar fat fraction in the interior ROI and phosphocreatine recovery time constant are observed at baseline $_{1}$ and baseline ${ }_{2}$, while reduced values are observed postintervention. DPN, diabetic peripheral neuropathy; ROI, region of interest.

region included all tissue within the muscle fascia except the tibia, and fibula (see figure 1, bottom row). All region of interests (ROIs) were eroded by $4 \mathrm{~mm}$ to minimize contamination from neighboring tissue. The proton relaxation values were measured in the GM, GL, and SOL in four slices $(16 \mathrm{~mm})$.

Tractography was performed in manually defined TN ROIs using MRTrix3 software. ${ }^{38}$ A threshold was subsequently applied to exclude voxels outside the central 37 slices or whose intensity was lower than $50 \%$ of the maximum in the tract. The mean ADC and FA were calculated in the resulting tracts.

To determine the phosphocreatine recovery time constant $\tau$ we normalized the signal amplitude such that its value at rest was 1 and fitted the signal after plantar flexion to a monoexponential model:

$$
P C r(t)=P C r_{0}\left(1-e^{-t / \tau}\right)+1-P C r_{0},
$$

where $P C r_{0}$ is phosphocreatine depletion immediately following plantar flexion. Owing to the $6 \mathrm{~s}$ temporal resolution of the measurement, cases with $P C r_{0}<15 \%$ were omitted since $\tau$ could not be fitted with confidence.

The paired, two-tailed Student's t-test was used to determine the statistical significance between: baseline ${ }_{1}$ and baseline $e_{2}$ MRI, baseline ${ }_{2}$ and postintervention MRI, and preintervention and postintervention clinical and diabetic markers. The Spearman rank correlation coefficient $\left(r_{\mathrm{s}}\right)$ was used to assess the association between 
Table 1 Participant characteristics, intervention compliance, and clinical tests $(n=20)$

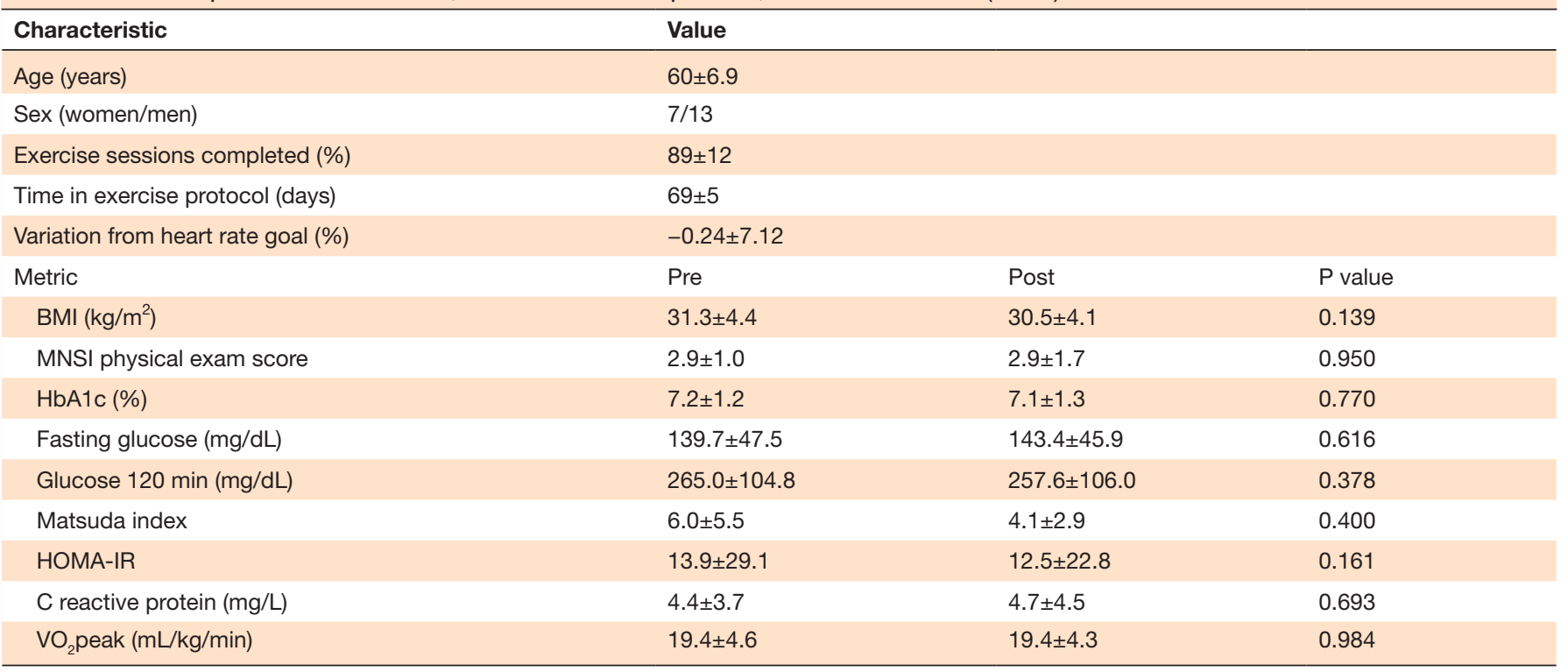

Pre indicates preintervention and post indicates postintervention. $\mathrm{P}$ indicates statistical differences between preintervention and postintervention values (paired, two-tailed Student's t-test).

Two $\mathrm{VO}_{2}$ peak data sets were excluded due to scheduling and technical difficulties.

BMI, body mass index; HbA1c, glycosylated hemoglobin; HOMA-IR, homeostasis model assessment of insulin resistance; MNSI, Michigan Neuropathy Screening Instrument.

changes in MRI variables (expressed as per cent change between baseline ${ }_{2}$ and postintervention) and changes in diabetic markers (expressed as per cent change between preintervention and postintervention). Statistical significance was set at $\mathrm{p}<0.05$, and all tests are reported without multiple comparison correction and without regard to sex due to the exploratory nature of the study.

\section{RESULTS}

Thirty-four individuals were enrolled in the study. Seven were unable to complete the study due to the COVID-19 outbreak and seven withdrew prior to completion for the following reasons: one individual experienced ventricular ectopy with reduced systolic blood pressure during the treadmill $\mathrm{VO}_{2}$ peak test and was deemed unfit for exercise, two underwent surgery, two cited personal reasons and two provided no explanation.

Table 1 lists participant characteristics and exercise intervention compliance for the 20 individuals that completed the study; those individuals attended $89 \% \pm 12 \%$ of the 30 prescribed intervention sessions and achieved the desired heart rate to within $1 \%$. Medications prescribed by participants' physicians were maintained during the study: 16 of 20 participants used metformin, 14 used statins, 9 used insulin, and 1 used beta-blockers. The metformin, statin, and beta-blocker dosages were maintained throughout the study. In one participant, insulin was discontinued between baseline ${ }_{2}$ and postintervention. In another participant, insulin dosage was reduced from 200 to 100 units between baseline ${ }_{1}$ and baseline . .

Table 1 lists clinical and diabetic markers before and after 10-week intervention. No significant change was observed in BMI, MNSI score, $\mathrm{VO}_{2}$ peak, and blood markers related to diabetic risk $(p>0.1)$. Due to technical developments being carried out during the study, relaxation time measurements are reported only at baseline $_{2}$ and postintervention in a subset of 12 subjects, 8 of which were included in a previous study. ${ }^{35}$ No changes were observed in the clinical and diabetic markers in the 12-participant subset ( $p>0.1$ ) (online supplemental table 1).

Figures 1 and 2 show fat fraction maps, phosphocreatine signal plots, and relaxation time maps in a participant in their 50s. Table 2 summarizes MRI measurements from the cohort. The following data sets were excluded: one fat fraction case and two DTI cases due to corrupt MRI files, one DTI case due to patient motion that prevented tractography, and five phosphocreatine cases due to less than $15 \%$ depletion. No significant change between baseline $_{1}$ and baseline ${ }_{2}$ was observed in fat fraction in the leg interior or in individual muscle ROIs $(\mathrm{p}>0.4)$. There was a trend toward significant fat fraction reduction after intervention in the leg interior ROI: $-2.6 \pm 6.4 \%(p<0.1)$, while insignificant change was observed in individual calf muscles $(\mathrm{p}>0.5)$. T1 $(-2.3 \pm 3.0 \%)$, T2 $(-3.5 \pm 6.4 \%)$, and $\mathrm{T} 1 \rho(-4.1 \pm 7.7 \%)$ changes were observed in GM $(\mathrm{p}<0.1)$, and T2 changes were observed in GL $(-4.6 \pm 7.4 \%)$ and SOL $(-3.2 \pm 3.1 \%) \quad(\mathrm{p}<0.1)$. No changes in FA or ADC were observed between baseline ${ }_{1}$ and baseline ${ }_{2}(p>0.3)$ or between baseline ${ }_{2}$ and postintervention $(p>0.4)$. No change in phosphocreatine recovery time constant was observed between baseline $_{1}$ and baseline $_{2}(\mathrm{p}>0.5)$ or between baseline ${ }_{2}$ and postintervention ( $p>0.3$ ).

Table 3 lists pairs of MRI measurements and diabetic markers whose values changed between preintervention 


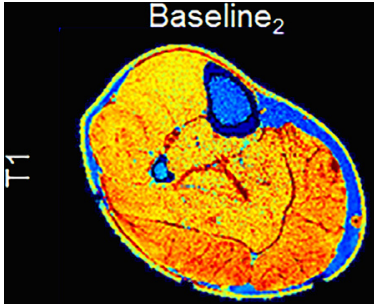

$\mathrm{GM} \mathrm{T1}=1025 \mathrm{~ms}$

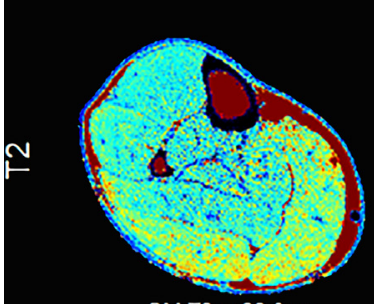

$\mathrm{GM} \mathrm{T2}=32.0 \mathrm{~ms}$

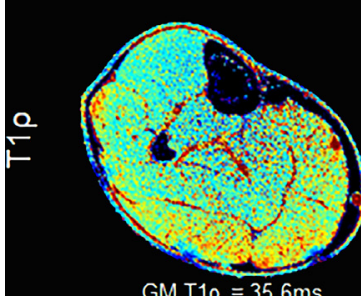

$\mathrm{GM} \mathrm{T} 1 \rho=35.6 \mathrm{~ms}$
Post-intervention

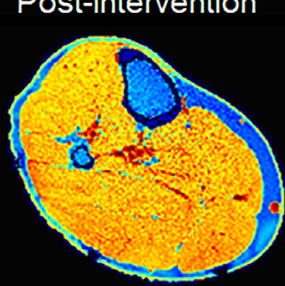

$\mathrm{GM} \mathrm{T} 1=942 \mathrm{~ms}$

0

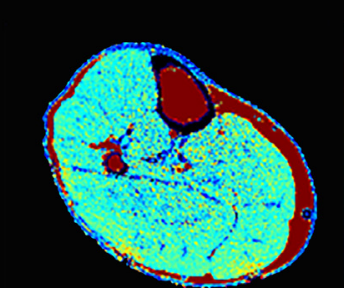

$\mathrm{GM} \mathrm{T} 2=26.1 \mathrm{~ms}$

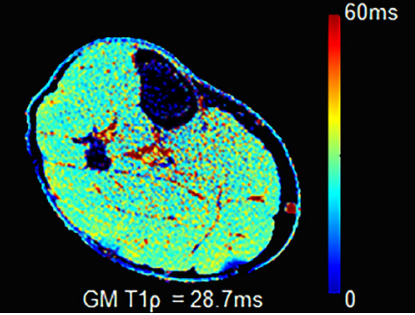

Figure 2 Relaxometry maps from the same subject as in figure 1 show reduced postintervention T1 (row 1), T2 (row 2), and $\mathrm{T} 1 \rho$ (row 3). Text overlays indicate average values in the gastrocnemius medialis (GM).

and postintervention in a correlated manner $(\mathrm{p}<0.05)$. We observed unexpected negative correlation between fat fraction in the interior ROI and $\mathrm{HbAlc}$ as well as unexpected positive correlations between relaxation times in the gastrocnemius muscles and $\mathrm{VO}_{2}$ peak. A significant positive association was found between MNSI physical exam score and SOL T2.

\section{DISCUSSION}

Recently modified therapeutic recommendations for individuals with DPN include physical exercise to improve muscle and nerve function. ${ }^{39}$ The early results from the combined resistance and aerobic exercise training intervention in this study show promise for improving skeletal muscle composition in individuals with DPN. The major findings are reductions in skeletal muscle adipose tissue and relaxation times in response to short-term exercise training.

No signficant difference in intramuscular fat fraction was observed after intervention in individual calf muscles. However, we observed a reduction in fat fraction in the global interior region of the leg. Improvement in muscle composition is important because DPN is associated with severely reduced muscle mass and increased interstitial fat and intramuscular fat. These skeletal muscle deficits are associated with loss of muscle strength, power and endurance as well as reduced physical function and ability to complete daily activities.

Relaxation times represent a range of muscle properties that reflect macromolecule makeup including proteins and membranes. Prior studies showed prolonged T2 and T1 $\rho$ in myositis, Duchenne muscular dystrophy, and DPN. ${ }^{24-28}$ Meanwhile T1 may also be prolonged in myopathic dermatomyositis. ${ }^{22}$ The reduced relaxation

\begin{tabular}{|c|c|c|c|c|c|c|c|c|c|}
\hline Metric & $\mathbf{N}$ & ROI & Baseline $_{1}$ & Baseline $_{2}$ & $\mathbf{P}_{\text {base }}$ & $\Delta_{\text {base }}(\%)$ & Post & $P$ value & $\Delta_{\text {post }}(\%)$ \\
\hline \multirow[t]{4}{*}{ FF (\%) } & 19 & Interior & $10.8 \pm 3.4$ & $10.8 \pm 3.7$ & 0.932 & $0.3 \pm 8.4$ & $10.5 \pm 3.3$ & 0.097 & $-2.6 \pm 6.4$ \\
\hline & 19 & GM & $8.6 \pm 4.6$ & $8.5 \pm 4.0$ & 0.733 & $1.7 \pm 11.3$ & $8.4 \pm 4.0$ & 0.564 & $-1.8 \pm 8.7$ \\
\hline & 19 & $\mathrm{GL}$ & $7.4 \pm 4.0$ & $7.6 \pm 3.8$ & 0.402 & $3.9 \pm 11.6$ & $7.5 \pm 3.9$ & 0.520 & $-2.5 \pm 12.0$ \\
\hline & 19 & SOL & $9.9 \pm 4.0$ & $9.8 \pm 4.1$ & 0.718 & $-0.5 \pm 7.7$ & $9.9 \pm 4.2$ & 0.855 & $0.0 \pm 9.5$ \\
\hline$\tau(\mathrm{s})$ & 15 & $\mathrm{GM}+\mathrm{GL}$ & $34.1 \pm 20.6$ & $35.4 \pm 21.8$ & 0.551 & $7.4 \pm 27.4$ & $32.3 \pm 21.7$ & 0.309 & $-4.6 \pm 30.2$ \\
\hline FA & 17 & $\mathrm{TN}$ & $0.34 \pm 0.06$ & $0.32 \pm 0.07$ & 0.319 & $-4.2 \pm 20.6$ & $0.33 \pm 0.05$ & 0.694 & $4.2 \pm 17.7$ \\
\hline $\operatorname{ADC}\left(\times 10^{3} \mathrm{~mm}^{2} / \mathrm{s}\right)$ & 17 & TN & $1.77 \pm 0.31$ & $1.76 \pm 0.20$ & 0.922 & $2.0 \pm 20.4$ & $1.81 \pm 0.19$ & 0.463 & $3.9 \pm 16.6$ \\
\hline \multirow[t]{3}{*}{$\mathrm{T} 1(\mathrm{~ms})$} & 12 & GM & & $940.9 \pm 43.3$ & & & $918.3 \pm 20.0$ & 0.023 & $-2.3 \pm 3.0$ \\
\hline & 12 & $\mathrm{GL}$ & & $947.5 \pm 42.4$ & & & $935.0 \pm 18.7$ & 0.274 & $-1.2 \pm 3.8$ \\
\hline & 12 & SOL & & $938.3 \pm 34.4$ & & & $935.7 \pm 23.6$ & 0.727 & $-0.2 \pm 2.7$ \\
\hline \multirow[t]{3}{*}{$\mathrm{T} 1 \rho(\mathrm{ms})$} & 12 & GM & & $31.0 \pm 3.7$ & & & $29.6 \pm 1.8$ & 0.078 & $-4.1 \pm 7.7$ \\
\hline & 12 & $\mathrm{GL}$ & & $30.0 \pm 4.5$ & & & $29.1 \pm 2.0$ & 0.365 & $-1.8 \pm 10.6$ \\
\hline & 12 & SOL & & $31.7 \pm 3.0$ & & & $31.6 \pm 2.8$ & 0.974 & $0.2 \pm 7.3$ \\
\hline \multirow[t]{3}{*}{ T2 (ms) } & 12 & GM & & $25.3 \pm 2.6$ & & & $24.2 \pm 1.2$ & 0.095 & $-3.5 \pm 6.4$ \\
\hline & 12 & $\mathrm{GL}$ & & $26.6 \pm 2.7$ & & & $25.2 \pm 1.8$ & 0.055 & $-4.6 \pm 7.4$ \\
\hline & 12 & SOL & & $23.9 \pm 1.3$ & & & $23.1 \pm 1.4$ & 0.003 & $-3.2 \pm 3.1$ \\
\hline
\end{tabular}

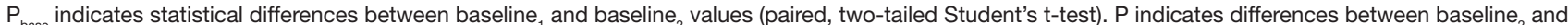
postintervention values. $P$ values in bold type indicate statistically significant differences or trends toward significance $(p<0.1)$.

ADC, apparent diffusion coefficient; $\Delta_{\text {base }}$, percentage change between baseline ${ }_{1}$ and baseline ${ }_{2}$ values; FA, fractional anisotropy; FF, fat fraction; GL, gastrocnemius lateralis; GM, gastrocnemius medialis; $\Delta_{\text {post }}$, percentage change between baseline ${ }_{2}$ and postintervention values; post, postintervention; ROI, region of interest; $S O L$, soleus; TN, tibial nerve. 
Table 3 Significant associations $(\mathrm{p}<0.05)$ between preintervention and postintervention percentage based changes in MRI and clinical variables

\begin{tabular}{|c|c|c|c|c|c|}
\hline MRI measurement & ROI & Clinical measurement & $\mathbf{N}$ & $r_{s}$ & $P$ value \\
\hline $\mathrm{FF}$ & Interior & $\mathrm{HbA1c}$ & 19 & -0.459 & 0.048 \\
\hline $\mathrm{T} 1$ & GM & $\mathrm{VO}_{2}$ peak & 11 & 0.646 & 0.037 \\
\hline $\mathrm{T} 1 \rho$ & GM & $\mathrm{VO}_{2}$ peak & 11 & 0.800 & 0.005 \\
\hline $\mathrm{T} 2$ & $\mathrm{GL}$ & $\mathrm{VO}_{2}$ peak & 11 & 0.627 & 0.044 \\
\hline $\mathrm{T} 2$ & SOL & MNSI physical exam score & 12 & 0.587 & 0.045 \\
\hline
\end{tabular}

FF, fat fraction; GL, gastrocnemius lateralis; GM, gastrocnemius medialis; HbA1c, glycosylated hemoglobin; MNSI, Michigan Neuropathy Screening Instrument; $\mathrm{ROI}$, region of interest; SOL, soleus.

times observed in this study following exercise intervention suggest a reduction of pathologic conditions in the muscle. We found the greatest $\mathrm{T} 1$ and $\mathrm{T} 1 \rho$ reduction in GM, while GL and SOL showed less change. This finding could be related to increased GM recruitment during calf exercises prescribed in the intervention of compared with GL and SOL. Interestingly, diabetic myopathy is more likely to be associated with GM deterioration than other calf muscles. ${ }^{40}$ While it has been suggested that skeletal muscle relaxation times may be entangled with adiposity, we found insignificant change in fat infiltration in GM, GL, and SOL. Thus, reduced relaxation times are more likely to represent atrophy reversal and muscle remodeling than adipose reduction, consistent with the finding of unchanged intramuscular fat within the calf muscles.

The lack of change in ADC, FA, and PCr recovery time constant indicates that TN microstructure and muscle energetics did not change to the extent necessary for detection with MRI. The potential disconnect between muscle composition and nerve remodeling and metabolic function suggests that more research is needed to explore whether exercise dosage or type can be modified to drive a response, to potentially determine the point at which nerve damage or metabolic dysfunction cannot be reversed in DPN, and whether DTI and ${ }^{31} \mathrm{P}-\mathrm{MRI}$ are sensitive enough to track therapy response. While the phosphocreatine recovery time constant following brief exercise directly probes muscle oxidative capacity, which cannot currently be accessed with proton MRI, the experiment is susceptibile to patient compliance. In our experience, some patients had difficulty following the pedal flexion acoustic cue due to competing noise from MRI gradient switching and were separately predisposed to performing inadequate or inconsistent flexion required for phosphocreatine depeletion. Other aspects such as epigenomic factors ${ }^{41}$ and medication use ${ }^{42} 43$ have been found to impact exercise response and also deserve further consideration. In parallel, the lack of change in MNSI, $\mathrm{VO}_{2}$ peak, and blood markers related to diabetic risk may be related to participants' relatively mild neuropathy and low baseline HbA1c, leaving little room for improvement.

One of the strengths of this study is the use of quantitative MRI. The Dixon-based method quantifies fat fraction with robustness against magnetic field heterogeneity, which can confound methods that, for example, rely on pixel intensity thresholds to distinguish between fat and muscle in a binary manner. Magnetic resonance fingerprinting is similarly robust against system imperfections and allows simultaneous quantitative $\mathrm{T} 1, \mathrm{~T} 1 \rho$, and $\mathrm{T} 2$ mapping that do not require subjective interpretation typical of 'contrast-weighted' images. Together, the techniques provide the means to monitor important diabetic markers during treatment.

One of the study limitations is lack of a control cohort, which makes it unclear whether the effects of exercise would be observed in matched BMI individuals without DPN. Indeed, weight and adipose reduction has been reported in overweight and obese individuals with T2DM, but without neuropathy, following 1-year diet and exercise intervention. ${ }^{44}$ Others have shown promising improvements in pain interference, vascular health, and neuropathic symptoms in individuals with DPN following 16-week ${ }^{2045}$ and 12-week exercise interventions. ${ }^{46}$ While there is no consensus on an optimal exercise regimen, ${ }^{47}$ our study suggests that adipose infiltration and muscle composition improve within 10 weeks. We acknowledge that the small sample size is a limitation and point out that multiple comparison corrections were not performed, which increase the possibility of false correlations between changes in MRI and clinical measurements (table 3). Another consideration concerning associations in table 3 is that MRI measurements are localized, whereas $\mathrm{VO}_{2}$ peak and HbAlc are global fitness metrics. Nonetheless, these exploratory associations merit further study.

An additional potential limitation is the heterogeneous nature of the cohort in which MNSI physical exam scores ranged from 1 to 7 . A natural extension of this work will be to relate change in clinical measures such as muscle strength to MRI measurements in a randomized controlled trial with a larger cohort of BMI-matched individuals with a range of DPN severity. A final limitation is that dietary factors were not tracked, which could contribute to posintervention changes detected with MRI.

\section{CONCLUSION}

Individuals with long-standing diabetes are at high risk for wide-ranging musculoskeletal complications such as pain, plantar ulcers, Charcot arthropathy, and amputations. Effective interventions are essential to reverse 
musculoskeletal complications in DPN, while quantitative markers are needed to monitor patient response. Others have shown that supervised exercise intervention improves glycemic control, reduces inflammation, and improves muscle strength and quality in type 2 diabetes. The results in this work used quantitative MRI to show that supervised exercise intervention reduced adiposity and relaxation times in the leg muscles in individuals with DPN. The results of a larger cohort may allow further assessment of improvements in muscle composition and potential associations between local MRI measurements and global measures of fitness, while interventions can be extended beyond the 10-week period tested here in attempt to instigate mitochondrial and nerve adaptations.

\section{Author affiliations}

'Department of Radiology, New York University Grossman School of Medicine, New York City, New York, USA

${ }^{2}$ Department of Radiology, Michigan State University, East Lansing, Michigan, USA ${ }^{3}$ Department of Psychiatry, New York University Grossman School of Medicine, New York City, New York, USA

${ }^{4}$ Department of Psychiatry, Nathan S Kline Institute for Psychiatric Research, Orangeburg, New York, USA

${ }^{5}$ Department of Osteopathic Medicine, New York Institute of Technology, Old Westbury, New York, USA

${ }^{6}$ Sports Performance Center, New York University Langone Health, New York City, New York, USA

${ }^{7}$ Department of Orthopedic Surgery, New York University Grossman School of Medicine, New York City, New York, USA

${ }^{8}$ Department of Physical Therapy, Rehabilitation Science, and Athletic Training, University of Kansas Medical Center, Kansas City, Kansas, USA

${ }^{9}$ Department of Physical Therapy, New York University, New York City, New York, USA

Acknowledgements The authors acknowledge Oleksandr Khegai for software development and study coordinators Amanda Ho, Shannon Haas, Elizabeth Yakubova, Magdalena Palac, Katherine Medina, Simone Rainey, Charles Morton, and Liz Calderon for recruiting participants, performing MNSI tests, collecting blood samples, carrying out exercise intervention procedures, and performing statistical analyses, and Aaron $\mathrm{R}$ Chidakel for supervising participant safety.

Contributors PP and SR conceptualized the study. AS, ND, SB, SR, and RB curated and analyzed the data. PP, SR, RRR, and RB acquired financial support for the project. AS, SR, HM, and RB conducted the experiments. PP, JMS, AS, SB, RRR, and RB designed the MRI protocol. SR and PMK designed the 10-week intervention program. SR and RB administered and supervised the study. AC and KJM assisted with patient recruitment. AS, SB, PP, RRR, and RB developed MRI software. All authors wrote and edited the manuscript. RB is the guarantor of this work and, as such, had full access to all the data in the study and takes responsibility for the integrity of the data and the accuracy of the data analysis.

Funding This work was supported in part by NIH grants R01 DK114428, R01 AR068966, R01 DK106292, R01 AR076985, and T35 DK007421, and was performed under the rubric of the Center for Advanced Imaging Innovation and Research (CAI2R, www.cai2r.net), a NIBIB Biomedical Technology Resource Center (NIH P41 EB017183).

Competing interests The authors declare that they have no competing interests. PP is currently employed by Regeneron Pharmaceuticals, Inc and declares no conflict of interest regarding the content of this manuscript.

Patient consent for publication Not required.

Ethical approval The study was conducted in accordance with Good Clinical Research Practice guidelines and the Declaration of Helsinki, was approved by New York University Langone Health's Institutional Review Board (\#18-00527), and all subjects provided informed written consent.

Provenance and peer review Not commissioned; externally peer reviewed.
Data availability statement Data are available upon reasonable request. The datasets generated during and/or analyzed during the current study are available from the corresponding author upon reasonable request.

Supplemental material This content has been supplied by the author(s). It has not been vetted by BMJ Publishing Group Limited (BMJ) and may not have been peer-reviewed. Any opinions or recommendations discussed are solely those of the author(s) and are not endorsed by BMJ. BMJ disclaims all liability and responsibility arising from any reliance placed on the content. Where the content includes any translated material, BMJ does not warrant the accuracy and reliability of the translations (including but not limited to local regulations, clinical guidelines, terminology, drug names and drug dosages), and is not responsible for any error and/or omissions arising from translation and adaptation or otherwise.

Open access This is an open access article distributed in accordance with the Creative Commons Attribution Non Commercial (CC BY-NC 4.0) license, which permits others to distribute, remix, adapt, build upon this work non-commercially, and license their derivative works on different terms, provided the original work is properly cited, appropriate credit is given, any changes made indicated, and the use is non-commercial. See: http://creativecommons.org/licenses/by-nc/4.0/.

ORCID iD

Ryan Brown http://orcid.org/0000-0002-2683-712X

\section{REFERENCES}

1 Centers for Disease Control and Prevention. National diabetes statistics report. Atlanta, GA; 2020.

2 Tesfaye S, Selvarajah D. Advances in the epidemiology, pathogenesis and management of diabetic peripheral neuropathy. Diabetes Metab Res Rev 2012;28 Suppl 1:8-14.

3 Boulton AJM, Vinik Al, Arezzo JC, et al. Diabetic neuropathies: a statement by the American diabetes association. Diabetes Care 2005;28:956-62.

4 Bittel DC, Bittel AJ, Tuttle LJ, et al. Adipose tissue content, muscle performance and physical function in obese adults with type 2 diabetes mellitus and peripheral neuropathy. $J$ Diabetes Complications 2015;29:250-7.

5 Cheuy VA, Hastings MK, Commean PK, et al. Muscle and joint factors associated with forefoot deformity in the diabetic neuropathic foot. Foot Ankle Int 2016;37:514-21.

6 Hilton TN, Tuttle LJ, Bohnert KL, et al. Excessive adipose tissue infiltration in skeletal muscle in individuals with obesity, diabetes mellitus, and peripheral neuropathy: association with performance and function. Phys Ther 2008;88:1336-44.

7 Boulton AJM, Kempler P, Ametov A, et al. Whither pathogenetic treatments for diabetic polyneuropathy? Diabetes Metab Res Rev 2013;29:327-33

8 Vinik A, Ullal J, Parson HK, et al. Diabetic neuropathies: clinical manifestations and current treatment options. Nat Clin Pract Endocrinol Metab 2006;2:269-81.

9 Finnerup NB, Sindrup SH, Jensen TS. The evidence for pharmacological treatment of neuropathic pain. Pain 2010;150:573-81.

10 Sigal RJ, Kenny GP, Boulé NG, et al. Effects of aerobic training, resistance training, or both on glycemic control in type 2 diabetes: a randomized trial. Ann Intern Med 2007;147:357-69.

11 Colberg SR, Sigal RJ, Fernhall B, et al. Exercise and type 2 diabetes: the American college of sports medicine and the American diabetes association: joint position statement. Diabetes Care 2010;33:e147-67.

12 Sigal RJ, Kenny GP, Wasserman DH, et al. Physical activity/exercise and type 2 diabetes: a consensus statement from the American diabetes association. Diabetes Care 2006;29:1433-8.

13 Singleton JR, Smith AG, Marcus RL. Exercise as therapy for diabetic and prediabetic neuropathy. Curr Diab Rep 2015;15:120.

14 Jahantigh Akbari N, Hosseinifar M, Naimi SS, et al. The efficacy of physiotherapy interventions in mitigating the symptoms and complications of diabetic peripheral neuropathy: a systematic review. J Diabetes Metab Disord 2020;19:1995-2004.

15 Kluding PM, Pasnoor M, Singh R, et al. Safety of aerobic exercise in people with diabetic peripheral neuropathy: single-group clinical trial. Phys Ther 2015;95:223-34.

16 Kluding PM, Pasnoor M, Singh R, et al. The effect of exercise on neuropathic symptoms, nerve function, and cutaneous innervation in people with diabetic peripheral neuropathy. $J$ Diabetes Complications 2012;26:424-9.

17 Fisher MA, Langbein WE, Collins EG, et al. Physiological improvement with moderate exercise in type II diabetic neuropathy. Electromyogr Clin Neurophysiol 2007;47:23-8. 
18 Dixit S, Maiya AG, Shastry BA. Effect of aerobic exercise on peripheral nerve functions of population with diabetic peripheral neuropathy in type 2 diabetes: a single blind, parallel group randomized controlled trial. J Diabetes Complications 2014;28:332-9.

19 Kruse RL, Lemaster JW, Madsen RW. Fall and balance outcomes after an intervention to promote leg strength, balance, and walking in people with diabetic peripheral neuropathy: "feet first" randomized controlled trial. Phys Ther 2010;90:1568-79.

20 Yoo M, D'Silva LJ, Martin K, et al. Pilot study of exercise therapy on painful diabetic peripheral neuropathy. Pain Med 2015;16:1482-9.

21 Bäumer P, Pham M, Ruetters M, et al. Peripheral neuropathy: detection with diffusion-tensor imaging. Radiology 2014;273:185-93.

22 Park JH, Olsen NJ, King L, et al. Use of magnetic resonance imaging and P-31 magnetic resonance spectroscopy to detect and quantify muscle dysfunction in the amyopathic and myopathic variants of dermatomyositis. Arthritis Rheum 1995;38:68-77.

23 Vandenborne K, McCully K, Kakihira H, et al. Metabolic heterogeneity in human calf muscle during maximal exercise. Proc Natl Acad Sci U S A 1991;88:5714-8.

24 Virta A, Komu M, Lundbom N, et al. Low field T1rho imaging of myositis. Magn Reson Imaging 1998;16:385-91.

25 Huang Y, Majumdar S, Genant HK, et al. Quantitative MR relaxometry study of muscle composition and function in duchenne muscular dystrophy. J Magn Reson Imaging 1994;4:59-64.

26 Fujino H, Kobayashi T, Goto I, et al. Magnetic resonance imaging of the muscles in patients with polymyositis and dermatomyositis. Muscle Nerve 1991;14:716-20.

27 Bus SA, Yang QX, Wang JH, et al. Intrinsic muscle atrophy and toe deformity in the diabetic neuropathic foot: a magnetic resonance imaging study. Diabetes Care 2002;25:1444-50.

28 Stouge A, Khan KS, Kristensen AG, et al. MRI of skeletal muscles in participants with type 2 diabetes with or without diabetic polyneuropathy. Radiology 2020;297:608-19.

29 Peng X-G, Wang Y, Zhang S, et al. Noninvasive assessment of age, gender, and exercise effects on skeletal muscle: Initial experience with $T_{1} \rho \mathrm{MRI}$ of calf muscle. J Magn Reson Imaging 2017;46:61-70.

30 Herman WH, Pop-Busui R, Braffett BH, et al. Use of the Michigan neuropathy screening instrument as a measure of distal symmetrical peripheral neuropathy in type 1 diabetes: results from the diabetes control and complications Trial/Epidemiology of diabetes interventions and complications. Diabet Med 2012;29:937-44.

31 Fletcher GF, Ades PA, Kligfield P, et al. Exercise standards for testing and training. Circulation 2013;128:873-934.

32 Brown R, Khegai O, Parasoglou P. Magnetic resonance imaging of phosphocreatine and determination of bold kinetics in lower extremity muscles using a dual-frequency coil array. Sci Rep 2016;6:30568.

33 Liu C-Y, McKenzie CA, Yu H, et al. Fat quantification with IDEAL gradient echo imaging: correction of bias from T(1) and noise. Magn Reson Med 2007;58:354-64.
34 Tsao J, Jiang Y. Hierarchical ideal: robust water-fat separation at high field by multiresolution field map estimation. Proc Int/ Soc Magn Reson Med 2008;16:653 https://www.semanticscholar. org/paper/Hierarchical-IDEAL-\%E2\%80\%93-robust-water-fatseparation-at-Tsao-Jiang/b99b2fd0679866b7448d870ce876748a 2caefd $4 b$

35 Sharafi A, Medina K, Zibetti MVW. Simultaneous T1, T2, and T1 $\rho$ relaxation mapping of the lower leg muscle with $\mathrm{Mr}$ fingerprinting. Magn Resonan Med 2021;00:1-10.

36 Khegai O, Madelin G, Brown R, et al. Dynamic phosphocreatine imaging with unlocalized $\mathrm{PH}$ assessment of the human lower leg muscle following exercise at 3T. Magn Reson Med 2018;79:974-80.

37 Naimon ND, Walczyk J, Babb JS, et al. A low-cost Mr compatible ergometer to assess post-exercise phosphocreatine recovery kinetics. MAGMA 2017;30:281-9.

38 Tournier J-D, Smith R, Raffelt D, et al. MRtrix3: a fast, flexible and open software framework for medical image processing and visualisation. Neuroimage 2019;202:116137.

39 Kluding PM, Bareiss SK, Hastings M, et al. Physical training and activity in people with diabetic peripheral neuropathy: paradigm shift. Phys Ther 2017;97:31-43.

40 Huang BK, Monu JUV, Doumanian J. Diabetic myopathy: MRI patterns and current trends. AJR Am J Roentgenol 2010;195:198-204.

41 Stephens NA, Brouwers B, Eroshkin AM, et al. Exercise response variations in skeletal muscle $\mathrm{PCR}$ recovery rate and insulin sensitivity relate to muscle epigenomic profiles in individuals with type 2 diabetes. Diabetes Care 2018;41:2245-54

42 Konopka AR, Laurin JL, Schoenberg HM, et al. Metformin inhibits mitochondrial adaptations to aerobic exercise training in older adults. Aging Cell 2019;18:e12880.

43 Walton RG, Dungan CM, Long DE, et al. Metformin blunts muscle hypertrophy in response to progressive resistance exercise training in older adults: a randomized, double-blind, placebocontrolled, multicenter trial: the masters trial. Aging Cell 2019;18:e13039.

44 Gallagher D, Heshka S, Kelley DE, et al. Changes in adipose tissue depots and metabolic markers following a 1-year diet and exercise intervention in overweight and obese patients with type 2 diabetes. Diabetes Care 2014;37:3325-32.

45 Billinger SA, Sisante J-FV, Alqahtani AS, et al. Aerobic exercise improves measures of vascular health in diabetic periphera neuropathy. Int J Neurosci 2017;127:80-5.

46 Gholami F, Nazari H, Alimi M. Cycle training improves vascular function and neuropathic symptoms in patients with type 2 diabetes and peripheral neuropathy: a randomized controlled trial. Exp Gerontol 2020;131:110799.

47 Xia T-W, Yang Y, Li W-H, et al. Different training durations and styles of tai chi for glucose control in patients with type 2 diabetes: a systematic review and meta-analysis of controlled trials. BMC Complement Altern Med 2019;19:63. 